ORIGINAL ARTICLE

\title{
Trauma calls: role of the general surgeon and CT scanning
}

\author{
R G Dattani, T Richards, C D Smith, T R Magee
}

Emerg Med J 2005;22:339-341. doi: 10.1136/emj.2004.017582

Background and objective: General surgeons are required in only a minority of trauma call cases to assess for abdominal injuries. Computed tomography (CT) accurately detects blunt abdominal injuries and may have replaced the need for general surgeons at trauma calls. This study evaluated the role of general surgeons at trauma calls and assessed use of $\mathrm{CT}$ in cases of suspected abdominal trauma.

Methods: (a) Eighteen month analysis of trauma calls at a district general hospital and (b) three month prospective study of all trauma cases presenting to A\&E.

See end of article for authors' affiliations

Results: (a) There were 73 trauma calls and the mechanism of injury in most cases was a road traffic accident (RTA). Most patients had orthopaedic and/or neurosurgical injuries. The general surgeons assessed 22 trauma call patients. Abdominal injury was excluded in 13 (four by clinical examination and nine following (T). (b) Forty three patients fulfilled the criteria for a trauma call and 14 trauma calls were made. The mechanism of injury in most was RTA and most had orthopaedic and/or neurosurgical injuries.

Correspondence to: Mr R Dattani, Royal Berkshire Hospital, London Road, Reading, RGI 1 5AN, UK; rdattani@doctors. org.uk The general surgeons assessed 10/43 potential trauma call patients, and abdominal injury was excluded in five (one by clinical examination and four following CT).

Conclusion: A\&E staff managed most trauma calls. Most patients did not require general surgical intervention. For penetrating injuries, presence of a general surgeon remained crucial. For blunt injuries

Accepted for publication 24 August 2004 CT was an important adjunct. These data suggest that general surgeons do not routinely have to attend all trauma calls but can be called if abdominal and/or vascular injuries are specifically suspected.

$\mathrm{T}$ he trauma team constitutes a crucial approach to the early management of seriously injured patients. Advanced Trauma and Life Support (ATLS) guidelines and recommendations of the Royal College of Surgeons of England state that a trauma team should include a general surgeon. ${ }^{12}$ However, only a minority of all trauma patients require assessment for abdominal and/or vascular injuries by a general surgeon, with even fewer requiring surgical intervention. ${ }^{3}$ Abdominal tenderness often is not predictive of abdominal injury in the multiply injured patient. ${ }^{4}$ The use of focused assessment with sonography for trauma (FAST) in the evaluation of blunt abdominal trauma can result in underdiagnosis of abdominal injuries. ${ }^{5}$ Computed tomography (CT) is both sensitive and specific in detecting blunt abdominal injuries, ${ }^{67}$ and increased availability of CT imaging may have replaced the need for a general surgeon at trauma calls.

We aimed to (a) evaluate the role of the general surgeon as a member of the trauma team and $(b)$ assess the use of CT imaging in cases of suspected abdominal trauma.

\section{METHODS}

We analysed all trauma calls made at the Royal Berkshire Hospital during an 18 month period (between October 2001 and March 2003), and followed this by a three month prospective study of all trauma cases presenting to the Accident and Emergency (A\&E) department. We assessed the mechanism of injury, presentation, diagnosis, radiological investigations, and management of all patients.

\section{Setting}

The Royal Berkshire Hospital (RBH) is a district general hospital in the UK, within $6.4 \mathrm{~km}$ (four miles) of the M4 motorway. It serves a population of approximately 500000. RBH A\&E deals with approximately 65000 new attendances per year. During office hours, A\&E staff are responsible for initial management of trauma patients. A trauma call is not always made. Following primary and secondary surveys, the

appropriate teams are alerted. During on-call hours, a trauma team is activated. Members of the trauma team are shown in box 1 .

The general surgical team do not routinely attend trauma calls but are contacted if $(a)$ there are multiple casualties expected or $(b)$ the orthopaedic registrar is unable to attend or $(c)$ abdominal and/or vascular injuries are specifically suspected. The trauma team is alerted if a patient fulfils a fixed set of criteria (box 2), which have been modified from those recommended by the Royal College of Surgeons of England and the British Orthopaedic Association. ${ }^{2}$

\section{RESULTS}

\section{Eighteen month analysis of trauma calls}

Between October 2001 and March 2003 there were 73 trauma calls. The mechanism of injury in most of the cases was an RTA (fig 1). The majority of patients had orthopaedic and/or neurosurgical injuries (table 1). The orthopaedic surgeons managed all the head and chest injuries.

General surgeons assessed 22 trauma call patients (30.1\%): abdominal injury was excluded in 13 patients $(17.8 \%$; four by clinical examination (5.5\%) and nine following a CT $(12.3 \%))$, and only nine patients $(12.3 \%)$ were admitted under the general surgeons. Seven patients (9.6\%) required operative surgical intervention: four for penetrating injuries (three abdominal stabbings, one laceration to axilla) and three for blunt trauma (splenic injury, liver injury, and oesophageal injury). Two patients $(2.7 \%)$ were admitted for observations. Both had superficial abdominal lacerations incurred by stabbing and both made an unremarkable recovery.

\section{Three month prospective study}

Between April and June 2003, 43 patients fulfilled the criteria for a trauma call (see box 2). Fourteen trauma calls $(32.6 \%)$

Abbreviations: $\mathrm{CT}$, computed tomography; $\mathrm{A} \& \mathrm{E}$, accident and emergency; RBH, Royal Berkshire Hospital; RTA, road traffic accident 
Box 1: Members of trauma team at the Royal

\section{Berkshire Hospital}

- A\&E consultant (during working hours)

- A\&E middle grade doctor (registrar or staff grade)

- Anaesthetic registrar

- Intensive care unit senior house officer (SHO)

- Orthopaedic SHO and registrar

- Two A\&E nurses

- Radiographer

- Porter

Box 2 Criteria for activating the trauma team at the Royal Berkshire Hospital

- Road traffic accident (RTA) involving fatality

- RTA where patient has been ejected from the vehicle

- RTA with vehicle roll over

- Fall from a height $>6 \mathrm{~m}$ (20 feet)

- Any gunshot wound

- Knife wound above the waist

- Hanging

- Drowning

- Major burns or explosion

- Electrocution

- Crush injury to the trunk or limbs

- A child pedestrian or cyclist hit by a vehicle

- Any trauma presenting with low blood pressure ( $<100 \mathrm{~mm} \mathrm{Hg}$ systolic), tachycardia (pulse >100/ $\mathrm{min})$, airway compromise, or signs of pneumothorax

were made. The mechanism of injury in most cases was an RTA (fig 2), and most of the injuries were orthopaedic and/or neurosurgical (table 2).

The general surgeons assessed $10(23.3 \%)$ of the 43 potential trauma call patients. Abdominal injury was excluded in five patients (11.6\%): one by clinical examination $(2.3 \%)$ and four following a CT $(12.3 \%)$. Only six patients $(14.0 \%)$ were admitted under the general surgeons: four patients $(9.3 \%)$ for observation (three superficial abdominal lacerations, one abdominal bruising) and two (4.7\%) required splenectomy.

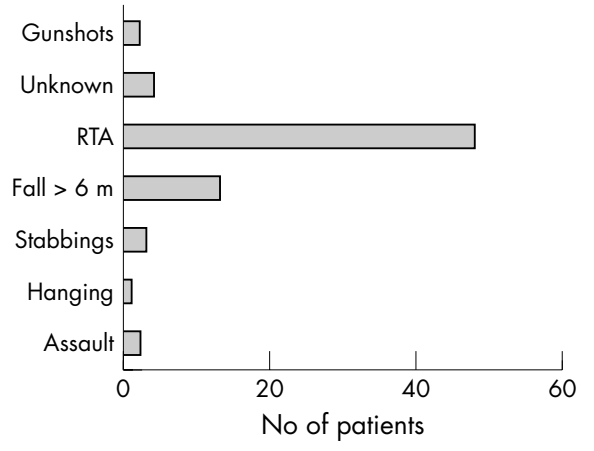

Figure 1 Mechanism of injury in 73 trauma call patients (October 2001-March 2003).
Table 1 Nature of injuries in trauma call patients (October 2001-March 2003)

\begin{tabular}{lc}
\hline & No $(\%)$ of patients \\
\hline Orthopaedic injuries & $19(26.0)$ \\
Neurosurgical injuries & $8(11.0)$ \\
Maxillofacial injuries & $2(2.7)$ \\
Combination of the above & $29(39.7)$ \\
General surgical & $9(12.3)$ \\
Fatal cardiac arrests & $6(8.2)$ \\
\hline
\end{tabular}

\section{DISCUSSION}

The trauma team approach to management of a seriously injured patient remains crucial for improving patient outcome. Currently, most of the trauma cases in our unit are managed by A\&E staff. This may reflect the availability of senior A\&E staff and high incidence of orthopaedic and/or neurosurgical injuries. Following initial assessment in A\&E, these patients are referred directly to orthopaedic surgeons. In the present study, although general surgeons assessed up to $30 \%$ of the patients, only a minority were admitted under their care. Despite absence of a general surgeon in the trauma team, no patient died from undiagnosed abdominal injury.

Twelve of 16 trauma call patients $(75 \%)$ and six of seven potential trauma call patients $(85.7 \%)$ had a CT scan for suspected abdominal trauma. Abdominal CT was positive in three of the twelve trauma call patients $(25 \%)$ and two of the six potential trauma call patients $(33 \%)$. These results are similar to those of a prospective multicentre study of 2299 patients with suspected blunt abdominal trauma. ${ }^{4}$ That study also showed that CT correctly predicted abdominal injuries in $99.63 \%$ of their patients and admission or observation was not necessary after a negative abdominal CT scan. In our study, all patients who had surgical intervention for blunt trauma had had a prior positive CT scan. No patients with a negative CT scan underwent an operation.

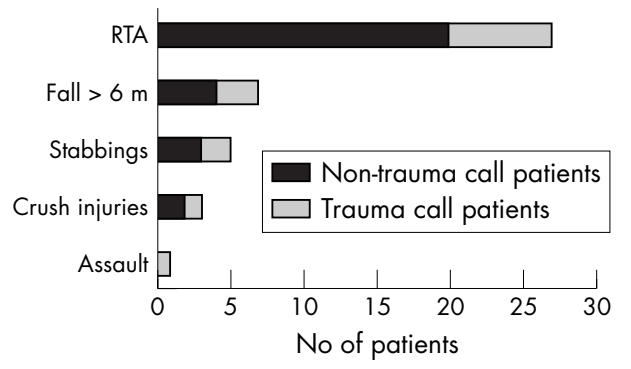

Figure 2 Mechanism of injury in 43 potential trauma calls (April-June 2003).

\begin{tabular}{ll}
\hline $\begin{array}{l}\text { Table } 2 \text { Patients fulfilling criteria for a trauma } \\
\text { call (April-June 2003) }\end{array}$ \\
\hline & No (\%) of patients \\
\hline Orthopaedic injuries & $17(39.5)$ \\
Neurosurgical injuries & $8(18.6)$ \\
Maxillofacial injuries & $3(7.0)$ \\
Chest injuries & $1(2.3)$ \\
Combination of the above & $7(16.3)$ \\
General surgical & $6(14.0)$ \\
Fatal cardiac arrests & $1(2.3)$ \\
\hline
\end{tabular}


In the case of penetrating injury $(8.2 \%$ of trauma call patients and $7.0 \%$ of potential trauma call patients), general surgical assessment was essential to determine if operative intervention was required. Most of the patients admitted under the general surgeons had a penetrating injury.

The low incidence of intra-abdominal and vascular injury was similar to the findings of the North Staffordshire Trauma Centre and the first report of the UK Major Trauma Outcome Study. ${ }^{38}$ This reflects the pattern of trauma seen in the UK where RTA is the commonest mechanism of injury, compared with the USA where penetrating injury can make up to $40 \%$ of trauma cases. ${ }^{3}$

\section{CONCLUSION}

In the present study, the majority of casualties generating a trauma call were managed by A\&E staff. Most patients did not require general surgical intervention. In penetrating injuries, presence of a general surgeon remained crucial. In blunt injuries, CT was an important adjunct to aid diagnosis. These data suggest that general surgeons do not routinely have to attend all trauma calls but can be called if abdominal and/or vascular injuries are specifically suspected.
Authors' affiliations

R G Dattani, T Richards, T R Magee, Department of General Surgery, Royal Berkshire Hospital, Reading, UK

C D Smith, Department of Orthopaedic Surgery, Royal Berkshire Hospital, Reading, UK

Competing interests: none declared

\section{REFERENCES}

1 American College of Surgeons. ATLS Handbook. Chicago, IL: ACS, 1990.

2 Royal College of Surgeons of England. Better Care for the Severely Injured. A Joint Report from the Royal College of Surgeons of England and the British Orthopaedic Association. London: RCS, 2000.

3 Bain IM, Kirby RM, Cook AL, et al. Role of the general surgeon in a British trauma centre. Br J Surg 1996;83:1248-51.

4 Livingstone $\mathrm{DH}$, Lavery RF, Passannante MR, et al. Admission or observation is not necessary after a negative abdominal computed tomographic scan in patients with suspected blunt abdominal trauma: results of a prospective, multi-institutional trial. J Trauma 1998;44:273-80.

5 Miller MT, Pasquale MD, Bromberg WJ, et al. Not so FAST. J Trauma 2003;54:52-9.

6 Jones TK, Walsh JW, Maull KI. Diagnostic imaging in blunt trauma of the abdomen. Surg Gynecol Obstet 1983;157:389-98.

7 Weishaupt D, Grozaj AM, Willmann JK, et al. Traumatic injuries: imaging of abdominal and pelvic injuries. Eur Radiol 2002;12:1295-311.

8 Yates DW, Woodford M, Hollis S. Preliminary analysis of the care of injured patients in 33 British hospitals; first report of the United Kingdom Major Trauma Outcome Study. BMJ 1992;305:737-40. 